\title{
Das zweite Opfer: Entwicklung eines Handlungsrahmens für den betriebsinternen Umgang mit Zwischenfällen
}

\author{
Die emotionale und gesundheitliche Belastungssituation von Ärztinnen und \\ Ärzten durch die Beteiligung an Zwischenfällen und Fehlern ist oft erheblich. \\ Die Stiftung für Patientensicherheit führt mit grosszügiger Unterstützung \\ durch die FMH ein Projekt durch, in dem ein praxisorientierter Handlungs- \\ rahmen für den betriebsinternen Umgang mit Zwischenfällen entwickelt wird. \\ Dabei werden Massnahmen und Empfehlungen erarbeitet, die den systema- \\ tischen und konstruktiven Umgang mit Fehlern und die Unterstützung der \\ Mitarbeitenden fördern sollen.
}

David Schwappacha,

Marc-Anton Hochreutener ${ }^{b}$

Stiftung für Patientensicherheit

a PD Dr., MPH,

Wissenschaftlicher Leiter

b Dr. med., Geschäftsführer

Korrespondenz:

Stiftung für Patientensicherheit

Asylstrasse 41

CH-8032 Zürich

Tel. 0432437621

schwappach@patientensicherheit.ch

\section{Hintergrund}

Die Bedeutung von Zwischenfällen und medizinischen Fehlern für die Belastungssituation von Ärztinnen und Ärzten ist international gut dokumentiert. In einer Kohortenstudie unter US-amerikanischen Assistenten in der Inneren Medizin berichteten 34\% der Befragten innerhalb des Untersuchungszeitraumes von etwa drei Jahren, mindestens einen bedeutsamen medizinischen Fehler gemacht zu haben. Die Beteiligung an einem Fehler war stark assoziiert mit einer nachfolgend reduzierten allgemeinen Lebensqualität, der starken Zunahme einer Burn-out-Symptomatik sowie einem etwa dreifach erhöhten Risiko für eine manifeste Depression und einer abnehmenden Empathie für Patienten [1]. Gleichzeitig mehrt sich die Evidenz, dass reduzierte Empathie, Depression und Burn-out-Symptomatik mit zukünftigen Fehlern assoziiert sind. Aktuelle Studien legen einen reziproken Zyklus nahe, in dem medizinische Fehler zu erheblicher persönlicher Belastung führen, die wiederum das Risiko für Fehler erhöht und damit die Patientensicherheit beeinflussen kann [1-4].

Eine Befragung von Assistenten in der Notfallmedizin zeigt, dass die Beteiligung an einem bedeutsamen Fehler mit hoher emotionaler Belastung verbunden ist [5]. Über die Hälfte der Befragten, die einen Fehler berichteten, fühlten sich in der Folge stark durch «Reue» $(68 \%)$, «Verzweiflung» (63\%), «Unzulänglichkeit» (58\%), «Frustration» $(55 \%)$ oder «Schuld» $(53 \%)$ belastet. Als Ur-

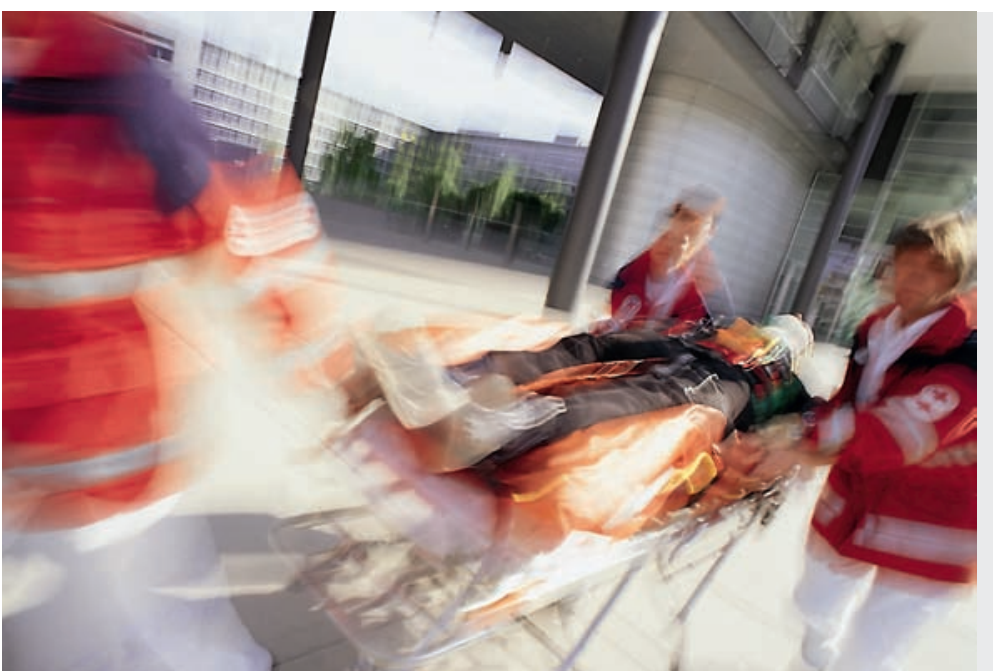

Arbeiten unter Druck: Medizinische Fehler haben nicht nur für Patienten nachteilige Folgen - sie belasten auch die involvierten Ärzte erheblich. 


\section{La deuxième victime: élaboration d'un cadre d'action pour la gestion interne des incidents critiques}

L'importance des incidents critiques et des erreurs médicales chez les médecins en situation de surcharge professionnelle est internationalement bien documentée. Etre à l'origine d'une faute, potentiellement suivie de conséquences graves pour le patient, entraîne fréquemment un sentiment durable de culpabilité chez l'auteur, ainsi qu'une réduction de la qualité de vie et une augmentation des risques de souffrir d'un burn-out, d'une dépression et d'un affaiblissement du sentiment d'empathie envers les patients. La Fondation pour la sécurité des patients se penche sur la problématique de la surcharge de travail des médecins sous l'angle des incidents critiques et lance un projet scientifiquement fondé et axé sur la pratique. Ce projet a pu voir le jour grâce au généreux soutien de la FMH. Un cadre d'action a été établi. II comprend des recommandations pour une gestion constructive et systématique des collaborateurs ayant provoqué des incidents critiques internes. Ce cadre d'action se fonde sur des données empiriques et scientifiques et est supervisé par un groupe d'experts nationaux. Cette base permettra l'élaboration de moyens auxiliaires et de documentation de même qu'un module de formation destinés aux fournisseurs de prestations. Les résultats et matériaux ainsi obtenus permettront de jeter les bases d'un soutien pratique visant à améliorer la situation des médecins concernés ainsi que les réactions face aux incidents critiques dans les établissements. Le projet fournit ainsi une contribution importante non seulement à la santé et à la qualité de travail des jeunes médecins, mais également des autres groupes professionnels. sachen für Fehler wurden vor allem intrinsische Gründe, insbesondere die mangelnde eigene Erfahrung, weniger aber extrinsische Gründe, wie eine mangelnde Supervision oder schlechte Arbeitsbedingungen, angegeben. Gerade junge Ärzte stehen in der Gefahr, sich in unangemessener Weise die Verantwortung für Fehler zuzuschreiben und «die Schuld bei sich zu suchen» [6]. Berichte aus den USA zeigen deutlich, dass die durch einen eigenen Fehler ausgelösten Emotionen eine universelle Erfahrung bei Ärzten sind, die oft eine dauerhafte «emotionale Narbe» zurücklassen [7]. Die Erfahrung eines Fehlers, der womöglich mit schweren Konsequenzen für den Patienten verbunden war, resultiert häufig in jahrelangen Schuldgefühlen, Scham und nicht selten in Veränderungen der ärztlichen Praxis [8]. Albert $\mathrm{Wu}$, ein international anerkannter Wissenschaftler auf dem Gebiet der Patientensicherheit, spricht daher auch von Ärzten als «second victim» $[9,10]$.

Diese Auswirkungen führen sicher in einer substantiellen Grössenordnung auch zum «Berufsausstieg vor dem Einstieg», d.h., dass junge Mediziner nach der Ausbildung nicht in die Patientenversorgung eintreten. Eine aktuelle Studie aus Dänemark zeigt, dass der Gedanke, einen Patienten schädigen zu können, bei 35\% der befragten jungen Ärzte dazu führt, dass sie «gele- gentlich oder sogar oft» darüber nachdenken, ihren Beruf aufzugeben [11]. Damit ist die Wirkung von unerwünschten Ereignissen und Fehlern auf die Situation von Ärzten auch von standespolitischer Bedeutung.

Als bedeutsamste Bedingungen, um mit der Erfahrung eigener Fehler umgehen zu lernen und sie bewältigen zu können, werden prioritär der Austausch mit Kollegen und die Unterstützung durch Führungspersonen genannt [6, 12]. Gleichzeitig werden Fehler nur sehr begrenzt auch intern und informell offengelegt $[11,13]$. Newman berichtet, dass paradoxerweise bei betroffenen Ärzten zwar einerseits der Wunsch nach Austausch besteht, gleichzeitig aber die Bereitschaft, dies Kollegen entgegenzubringen, eher gering ausgeprägt ist [12]. Dabei zeigt sich, dass ein «offenes Ohr» der Kollegen und Vorgesetzten häufig nicht ausreicht, sondern vielmehr ein System proaktiver und initiativer Ansprache erforderlich ist, z. B. das Berichten von eigenen Fehlern durch erfahrene Kollegen.

Der Umstand, dass es zu einem solchen hilfreichen Austausch zwischen Assistenten und erfahrenen Kollegen kommt, ist bis heute in vielen Fällen von einer mehr zufälligen Konstellation der beteiligten Persönlichkeiten abhängig. Systematische und institutionalisierte Unterstützung zum Umgang mit Fehlern wäre erforderlich, ist 
Beispielhaftes Zitat eines Assistenten aus der Interviewstudie von Engel et al. [6]

And in the case where it was my fault I was blessed with an attending who was diligent enough, who forced me to talk about it. Otherwise I would not have... And so I felt like I really was able to deal with that and put it behind me ... [Another case] just never got handled, it never got dealt with because the attending wasn't somebody I could approach.

aber selten. Die üblichen Fallbesprechungen und Rapporte werden zu wenig genutzt, um unerwünschte Ereignisse zu diskutieren [13, 14]. Insbesondere wird eine eindeutige und klare Sprache vermieden und selten die Chance genutzt, die Aufmerksamkeit gegenüber sicherheitsrelevanten Ereignissen zu schärfen.

Inwiefern diese Befunde übertragbar sind auf andere Berufsgruppen, ist zwar nicht genau bekannt, da es hierzu kaum Untersuchungen gibt. Trotzdem ist anzunehmen, dass bei anderen Berufsgruppen - wenn auch teilweise anders akzentuiert -ähnliche Problemstellungen bestehen.

Die Evidenz zeigt, dass Handlungsleitlinien für den systematischen und konstruktiven betriebsinternen Umgang mit Zwischenfällen dringend erforderlich sind und einen wichtigen Beitrag für die Unterstützung, Gesundheit und Arbeitsqualität insbesondere junger Ärzte, aber auch anderer Berufsgruppen leisten können. Ein solcher Handlungsrahmen ist in hohem Masse kulturabhängig. Daher muss den individuellen Bedürfnissen der Beteiligten, der Führungskräfte und Vorgesetzten ebenso wie den Spitälern und den betriebsinternen Organisationsstrukturen Rechnung getragen werden.

Neben der Offenlegung von Fehlern innerhalb des Betriebes hat auch die Offenlegung gegenüber Patienten einen «heilenden» und stressreduzierenden Effekt für die Beteiligten [15-18]. Die Stiftung für Patientensicherheit hat daher in Kooperation mit der Stiftung für Patientensicherheit in der Anästhesie erfolgreich umfas-

\section{Abbildung 1}

Fehlerbeteiligung und psychische, gesundheitliche Situation: ein reziproker Zyklus?

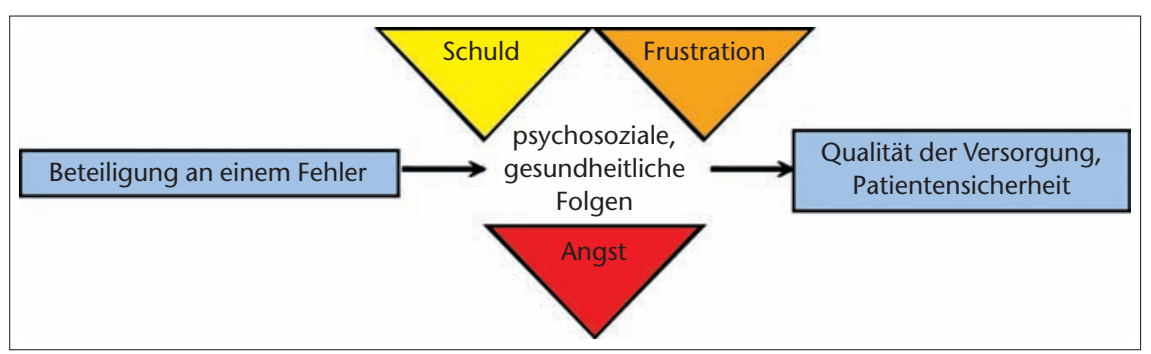

sende Handlungsempfehlungen für die angemessene Reaktion und Kommunikation nach dem Eintreten von Zwischenfällen gegenüber Patienten und Angehörigen entwickelt und breit lanciert [19].

Die Situation des von einem Zwischenfall betroffenen Personals («second victims») ist aber ebenfalls oft dramatisch. Die Stiftung für Patientensicherheit nimmt sich der Thematik der Belastung von Ärztinnen und Ärzten durch die Beteiligung an Zwischenfällen nun in einem wissenschaftlich fundierten und gleichzeitig praxisorientierten Projekt an. Die FMH ermöglicht mit ihrer grosszügigen Unterstützung die erfolgreiche Umsetzung dieses Projektes. Darüber hinaus leistet sie durch die Entsendung von Experten in die Expertengruppe auch wichtige inhaltliche Beiträge. Damit verknüpft sie in engagierter und zukunftsorientierter Weise die Interessen ihrer Mitglieder und des ärztlichen Berufsstandes mit der Sicherheit von Patienten.

\section{Das Projekt}

\section{Zielsetzung und Nutzen}

Das Projekt will einen Handlungsrahmen erstellen, der Empfehlungen für den konstruktiven und systematischen betriebsinternen Umgang mit Zwischenfällen gegenüber den Mitarbeitenden ausspricht. Dieser Handlungsrahmen soll in enger Ableitung aus empirischen, wissenschaftlichen Erkenntnissen und unter inhaltlicher Expertise und Beurteilung eines nationalen Expertengremiums entwickelt werden. Hierfür ist es notwendig, einerseits die internationale Evidenz zu berücksichtigen, andererseits auch neue empirische Daten für den nationalen Kontext zu generieren.

Die Ziele des Projektes sind:

- die Unterstützung der ärztlichen Mitarbeiter im Umgang mit Zwischenfällen;

- die Förderung der Sicherheitskultur - sowohl im Sinne der Aus-, Weiter- und Fortbildung als auch im Sinne des klinischen Risikomanagements. Hierzu gehört insbesondere die Förderung der aktiven und passiven Thematisierung von Zwischenfällen innerhalb der und zwischen den Hierarchieebenen. Damit wird auch ein wichtiger Beitrag für die Förderung der Meldebereitschaft geleistet (aktive Beteiligung an Meldesystemen).

Der Nutzen des Projektes liegt in einer Reduzierung des Belastungspotentials, das von Zwischenfällen und Fehlern ausgeht. Damit soll die Arbeitsund Berufszufriedenheit von Ärzten und die Reduzierung von Burn-out-Symptomatik, Mob- 
bing und Berufswechseln, gerade bei jungen Medizinern, gefördert werden.

Die Mitarbeitenden profitieren von der Verbreitung des Projektes und der Materialien/Schulungen sowohl in ihrer Rolle als Führungspersonen als auch in der Rolle als Betroffene. Kaderund Assistenzärzte erhalten mit Umsetzung des Projektes ein Bündel von Massnahmen, mit dem sie ihre Führungskompetenz spezifisch ausbauen können und ihre eigene und die Leistungsfähigkeit ihrer Mitarbeiter sichern und stärken können.

Das Projekt will (im Sinne der laufenden Arbeiten in der Schweizerischen Akademie der Medizinischen Wissenschaften [SAMW] zur Integration der Patientensicherheit in die Ausbildung) einen Beitrag zur Aus-, Weiter- und Fortbildung im Bereich Patientensicherheit leisten.

\section{Projektablauf}

Das Projekt ist auf fünf Module aufgebaut.

\section{Modul 1}

In einem ersten Schritt wurde die international verfügbare Evidenz zu effektiven Massnahmen, Strukturen und Prozessen des betriebsinternen Umganges mit Zwischenfällen und Beinahezwischenfällen im Rahmen eines systematischen Literaturreviews analysiert und zusammengefasst. Dabei wurde herausgearbeitet, welche Belastungen durch Zwischenfälle erwartet werden können und welche betriebsinternen Reaktionen, Strukturen und Interventionen besonders förderlich sind, um diese Belastungen zu reduzieren.

\section{Modul 2}

Da Organisations- und Führungskultur und damit auch die Anforderungen an betriebsinterne Reaktionen auf Zwischenfälle in hohem Mass kulturabhängig sind, sollen in einem zweiten Schritt neue empirische Erkenntnisse generiert werden, die die Situation in Schweizer Spitälern erfassen. Hierfür werden Fokusgruppen («Gruppendiskussionen») mit jungen Assistenten sowie Oberärzten und Führungskräften durchgeführt. Dabei geht es vor allem um die Identifikation von Faktoren, die eine positive Auseinandersetzung mit und Überwindung von Belastungssituationen erlauben und fördern. Auch die Suche nach individuellen «best practice»-Beispielen kann hilfreich sein. Bei der Umsetzung dieses qualitativen Forschungsansatzes ist es wichtig, die informellen, persönlichen und konkreten Erfahrungen zu erheben und nicht auf einer metakulturellen Ebene zu verbleiben. Der Einsatz qualitativer Verfahren ist sehr hilfreich, um die Komplexität der
Strukturen und Prozesse in Gesundheitseinrichtungen abbilden zu können [20]. Zudem sind qualitative Verfahren in der Lage, auch bisher nicht kognitiv reflektierte oder bewertete Erfahrungen zu erkennen und zu dokumentieren.

\section{Modul 3}

Die in den Modulen 1 und 2 gewonnenen empirischen Erkenntnisse werden in Form eines Thesenpapieres für die Praxisumsetzung zusammengefasst und in einem weiteren Schritt durch eine Expertengruppe in praxisrelevante Handlungsempfehlungen übersetzt. Dies erfolgt in einem iterativen Prozess, in dem wissenschaftliche Erkenntnisse mit den Erfahrungen und Umsetzungskenntnissen der Experten zusammengeführt werden. Die Entwicklung des Handlungsrahmens orientiert sich dabei stringent an der Zielsetzung, die Auswirkungen von Zwischenfällen und unerwünschten Ereignissen zu mildern und die betroffenen Individuen zu befähigen, mit dieser spezifischen Belastung umzugehen.

\section{Modul 4}

Die Projekterkenntnisse sollen direkten Nutzen bringen. Deswegen werden sie genutzt als Grundlage für die Entwicklung von praktischen Hilfsmitteln und Angeboten für die Leistungserbringer zur Umsetzung der erarbeiteten Empfehlungen. Dazu gehören insbesondere schriftliche Unterlagen, Kurzinformationen mit den wichtigsten Empfehlungen und Hinweisen sowie ein Schulungsmodul. Das Schulungsmodul wird einerseits für Kaderärzte mit Führungsverantwortung, andererseits für Assistenten entwickelt. Beide Module sollen jeweils einen Umfang von etwa $2 \times 2$ Stunden umfassen.

\section{Modul 5}

Im Rahmen einer ersten Umsetzung soll die Schulung in einem Pilotspital angeboten werden. Hierfür werden in einem motivierten Spital mit ausreichender Grösse und Mitarbeiterzahl sowohl das Kader- als auch das Assistentenmodul durchgeführt. Die Pilotschulung wird evaluiert, und die gewonnenen Erkenntnisse werden in einer Feedbackschleife verwendet, um etwaige Modifikationen am Schulungsmodul durchzuführen.

\section{Erwartete Ergebnisse}

Mit Abschluss des Projektes werden neben einer Aufarbeitung der internationalen Evidenz auch neue empirische Erkenntnisse für den Schweizer Kontext vorliegen. Die Erkenntnisse werden in Form von Thesen für die Praxisumsetzung aufbereitet und in einen praxisorientierten Handlungsrahmen und darin enthaltene Handlungs- 
empfehlungen übersetzt. Darauf aufbauend werden Hilfsmittel und Materialien für die Leistungserbringer zur Vermittlung und Umsetzung der Empfehlungen sowie ein Schulungsmodul erstellt. Die gesammelten Erkenntnisse und Materialien bilden die Grundlage, um praxisrelevante Umsetzungsaktivitäten zu initiieren und zu begleiten, mit dem Ziel, die Situation betroffener Ärzte und die Reaktion auf Zwischenfälle in den Betrieben zu verbessern.

\section{Literatur}

1 West CP, Huschka MM, Novotny PJ, Sloan JA, Kolars JC, Habermann TM, et al. Association of perceived medical errors with resident distress and empathy: a prospective longitudinal study. JAMA. 2006;296(9):1071-8.

2 Shanafelt TD, Bradley KA, Wipf JE, Back AL. Burnout and self-reported patient care in an internal medicine residency program. Ann Intern Med. 2002;136(5):358-67.

3 Shanafelt TD, West C, Zhao X, Novotny P, Kolars J, Habermann T, et al. Relationship between increased personal well-being and enhanced empathy among internal medicine residents. J Gen Intern Med. 2005;20(7):559-64

4 Fahrenkopf AM, Sectish TC, Barger LK, Sharek PJ, Lewin D, Chiang VW, et al. Rates of medication errors among depressed and burnt out residents: prospective cohort study. BMJ. 2008;336(7642): 488-91.

5 Hobgood C, Hevia A, Tamayo-Sarver JH, Weiner B, Riviello R. The influence of the causes and contexts of medical errors on emergency medicine residents' responses to their errors: an exploration. Acad Med. 2005;80(8):758-64

6 Engel KG, Rosenthal M, Sutcliffe KM. Residents' responses to medical error: coping, learning, and change. Acad Med. 2006;81(1):86-93.

7 Penson RT, Svendsen SS, Chabner BA, Lynch TJ, Jr., Levinson W. Medical mistakes: a workshop on personal perspectives. Oncologist. 2001;6(1):92-9.

8 Novack DH, Detering BJ, Arnold R, Forrow L, Ladinsky M, Pezzullo JC. Physicians' attitudes toward using deception to resolve difficult ethical problems. JAMA. 1989;261(20):2980-5.
9 Wu AW. Medical error: the second victim. BMJ. 2000;320(7237):726-7.

10 Wears RL, Wu AW. Dealing with failure: the aftermath of errors and adverse events. Ann Emerg Med. 2002;39(3):344-6.

11 Madsen MD, Ostergaard D, Andersen HB, Hermann N, Schioler T, Freil M. [The attitude of doctors and nurses towards reporting and handling errors and adverse events]. Ugeskr Laeger. 2006; 168(48):4195-200.

12 Newman MC. The emotional impact of mistakes on family physicians. Arch Fam Med. 1996;5(2):71-5.

13 Kaldjian LC, Jones EW, Rosenthal GE, Tripp-Reimer T, Hillis SL. An empirically derived taxonomy of factors affecting physicians' willingness to disclose medical errors. J Gen Intern Med. 2006;21(9): 942-48.

14 Pierluissi E, Fischer MA, Campbell AR, Landefeld CS. Discussion of medical errors in morbidity and mortality conferences. JAMA. 2003;290(21): 2838-42.

15 Gallagher TH, Levinson W. Disclosing harmful medical errors to patients: a time for professional action. Arch Intern Med. 2005;165(16):1819-24.

16 Gallagher TH, Waterman AD, Ebers AG, Fraser VJ, Levinson W. Patients' and physicians' attitudes regarding the disclosure of medical errors. JAMA. 2003;289(8):1001-7.

17 Schwappach DL, Koeck CM. What makes an error unacceptable? A factorial survey on the disclosure of medical errors. Int J Qual Health Care. 2004; 16(4):317-26.

18 Mazor KM, Simon SR, Gurwitz JH. Communicating With Patients About Medical Errors: A Review of the Literature. Arch Intern Med. 2004;164(15): 1690-7.

19 Stiftung für Patientensicherheit. Wenn etwas schief geht: Kommunizieren und Handeln nach einem Zwischenfall. Schriftenreihe Patientensicherheit Schweiz. Band 1. Zurich: Stiftung für Patientensicherheit; 2006.

20 Hoff TJ, Sutcliffe KM. Studying patient safety in health care organizations: accentuate the qualitative. Jt Comm J Qual Patient Saf. 2006; 32(1):5-15. 